\title{
Indirect Costs of Parasitism are Shaped by Variation in the Type of Immune Challenge and Food Availability
}

\author{
Pierre Bize • Romain Piault • Julien Gasparini • \\ Alexandre Roulin
}

Received: 19 April 2010/Accepted: 26 August 2010/Published online: 8 September 2010

(C) Springer Science+Business Media, LLC 2010

\begin{abstract}
Parasites can inflict indirect fitness costs to their hosts by eliciting costly immune responses. These costs depend on the type and amount of immunostimulants presented to the host immune system but also on the amount of resources available to fuel host immune responses. Here, we investigated how the relative costs of two different types of immune challenge are modulated by variation in food availability. We injected nestling tawny owls (Strix aluco) with either $10 \mu \mathrm{g}$ of phytohaemagglutinin (PHA) or $20 \mu \mathrm{g}$ of lipopolysaccharide (LPS), and subsequently raised them under two different food regimes (food-restricted vs. ad libitum). After controlling for food consumption, we found that LPS-injected nestlings lost more body mass than PHA-injected ones only when foodrestricted. We also found that body mass gain of owlets fed ad libitum decreased with the intensity of the skin swelling response against LPS, but not PHA. These experimental and correlative results suggest that nestling tawny owls suffered greater immune costs when treated with LPS than PHA, and that variation in the costs of two different types of immune challenge can be exacerbated under conditions of low food availability. Our study highlights the importance of taking into consideration the interplay between host immunity and nutrition in the study of indirect costs of parasitism.
\end{abstract}

Pierre Bize and Romain Piault contributed equally.

P. Bize $(\bowtie) \cdot$ R. Piault $\cdot$ J. Gasparini · A. Roulin Department of Ecology and Evolution, Biophore, University of Lausanne, 1015 Lausanne, Switzerland e-mail:Pierre.Bize@unil.ch

J. Gasparini

Laboratoire Ecologie et Evolution, Université Pierre et Marie Curie, CNRS UMR 7625, 7 quai St-Bernard, 75252 Paris, France
Keywords Condition-dependent · Food availability · Immunocompetence · Immunopathology · Tawny owl

\section{Introduction}

Parasites vary widely in their detrimental effects on host reproduction and survival. Evidence is accumulating that variation in the cost of parasitism is twofold, coming both from variation in host exploitation rates by parasites and from variation in the costs of host immune responses deployed against parasitic challenges. Indeed, despite the benefit of immune responses in fending off parasite infections, their activation and processing can limit or even impair other important host physiological functions such as growth, reproduction and body maintenance (Sheldon and Verhulst 1996; Zuk and Stoehr 2002; Stjernman et al. 2008). Hence, to understand the selection pressure exerted by parasites on their hosts, it is crucial to appraise not only which factors account for variation in host exploitation rates, referred to in the literature as 'direct' cost of parasitism (Frank 1996), but also factors responsible for variation in the costs of host immunity, referred to as 'indirect' cost of parasitism (Graham et al. 2005; Day et al. 2007).

There are at least two main sources of variation in the costs of host immune responses. First, immune costs vary with the type of immune pathways stimulated as well as with the magnitude and duration of elicited immune responses (Nossal 1983; Fair et al. 1999; Amat et al. 2007), with the most extreme cost being host death caused, for example, by excessive innate immune responses to high bacteria load (i.e. septic shock; Munford 2006) or virus titre (Kobasa et al. 2007). Second, evidence is 
accumulating that the expression of immune costs is condition-dependent (Moret and Schmid-Hempel 2000), with negative impacts of immunity on host fitness becoming evident mainly when food resources are limited. Several studies have investigated the costs associated with different antigens under standardized feeding conditions (e.g. Fair et al. 1999; Amat et al. 2007), or the costs associated with a single antigen under different feeding regimes (e.g. Jacot et al. 2004; Brzek and Konarzewski 2007). Although these studies confirm that costs of immunity vary among antigens and are condition-dependent, to our knowledge experiments are lacking where both antigen type and food availability are manipulated simultaneously. This experimental design is crucial to understand whether some parasites are more harmful to their hosts because they elicit more costly immune responses, and whether such differential indirect cost of parasitism is manifest under particular environmental conditions.

Here, we tested experimentally the prediction that two alternative immune challenges differentially affect offspring growth trajectory, and that such variation is exacerbated under poor food availability. To this end, we studied the growth of nestling tawny owls (Strix aluco) exposed to a two by two experimental design. Owlets were challenged with one of two immunostimulants injected in different doses (10 $\mu \mathrm{g}$ of lectin phytohaemagglutinin PHA vs. $20 \mu \mathrm{g}$ of lipopolysaccharide LPS), and subsequently fed ad libitum or food-restricted during the six following days. We then compared nestling body mass changes under these four experimental settings. In tawny owls, fledging body mass positively correlates with survival before dispersal (Overskaug et al. 1999), implying a selective advantage to nestlings that maintain body mass before leaving the nest. We choose PHA and LPS skin-swelling tests because of their amenability to fieldwork and because the immune responses induced by these two immunostimulants are well studied (Dantzer et al. 1998; Parmentier et al. 1998; Merino et al. 1999; Martin II et al. 2006; Tella et al. 2009). PHA is derived from plant beans of Phaseolus vulgaris and specifically induces T-cell division in vitro (Mire-Sluis et al. 1987). In birds, PHA has been shown to induce the infiltration of immune cell types from both the adaptive and innate components of the immune system at the injection site, and an increase in circulating T-cells in blood (Martin II et al. 2006; Tella et al. 2009). LPS is obtained from degenerated cell walls of gram-negative bacteria and is a mitogen for B lymphocytes (Skidmore et al. 1975), eliciting a specific antibody response in vivo even in extremely small amounts (Skidmore et al. 1975). As any immunogenic agents, both mitogens induce an acute-phase response, i.e. a series of metabolic adjustments that support immune responses (Klasing 1988). By injecting immunostimulants rather than inoculating different parasites we could assess the costs associated with different immune stimulations per se, irrespective of the costs imposed by parasite proliferation and concomitant damage. If low food availability exacerbates the differential cost of immune challenges, we predicted that the difference in growth rates between PHA- and LPS-injected nestlings should be greater in the food-restricted than in the ad libitum treatment, thus leading to a significant interaction between immune and food treatments.

\section{Methods}

Study Site and Species

The study was carried out between 2005 and 2010 in a population of tawny owls located in western Switzerland where 366 nest-boxes have been installed in an area of $911 \mathrm{~km}^{2}$. This bird preys upon a large number of animals including small rodents, birds and amphibians (Roulin et al. 2008). Breeding occurs from late February until early June. Only the female incubates the clutch, and half way through the rearing period she starts to help her partner collect food for the offspring. Eggs hatch asynchronously every 1-2 days leading to an age hierarchy among the siblings. Weighing $25-30 \mathrm{~g}$ on average at hatching, nestlings show fast body mass increment until they reach a plateau at 300-400 $\mathrm{g}$ around 23 days of age (unpublished data). From 21 to 25 days, young spend much time at nest entrance, and leave their nests 3-4 days later. At fledging, owlets have not yet fully grown their plumage and are barely able to fly. Thus, parents keep feeding their offspring outside the nest until they have reached a full adult size 60-75 days after fledging (Galeotti 2001). It has been shown elsewhere that nestling tawny owls have a peak of mortality due to starvation and predation immediately after fledging, and that heavier fledglings have a greater survival compared to light ones (Overskaug et al. 1999).

\section{Experimental Design}

In 2005 we visited nest-boxes to record clutch size (mean $\pm \mathrm{SE}$ based on the 80 experimental pairs: $4.20 \pm 0.11$ eggs, range: 2-6), hatching date (5th April \pm 1.2 days; 19th March-30th April), and brood size at hatching (3.61 \pm 0.12 nestlings; $2-5)$. Nestlings were banded with a numbered-aluminium ring to recognize them individually when $11.7 \pm 0.2$ day-old. When nestlings were $21.7 \pm 0.26$ days old, we injected subcutaneously in the wing web all siblings of 80 experimental nests either with LPS (serotype 055:B5; Sigma, L2880, Switzerland, $20 \mu \mathrm{g}$ diluted in $0.02 \mathrm{ml}$ of phosphate buffer 
saline) or with PHA (Sigma, L1668, Switzerland, $10 \mu \mathrm{g}$ diluted in $0.02 \mathrm{ml}$ of phosphate buffer saline). All the siblings in a brood where injected on the same day, and thus age at injection varies from 1 to 4 days among siblings due to hatching asynchrony. Subsequently, only one randomly chosen nestling per nest was considered in the present study (see below). Given that extra-pair young are rare in the tawny owl ( 1 out of 137 nestlings; Saladin et al. 2007), owlets involved in the present study were assumed all to be unrelated. We did not create a control group with owlets being injected with a saline solution (PBS) because our aim was to create two contrasting immune challenges (here that differ in both the dose and nature of the immunostimulant injected: $10 \mu \mathrm{g}$ PHA vs. $20 \mu \mathrm{g}$ LPS) rather than to study the cost of a particular immune challenge (see also Piault et al. 2009; Gasparini et al. 2009). By injecting both mitogens in the same way (i.e. subcutaneously), at the same location (i.e. in the wing web), and with the same amount of saline buffer, we tried to minimize variation between immune treatment groups in local inflammation as a direct result of tissue damage. We measured the thickness change at the injection site (a measure of the inflammatory response) $4.0 \pm 0.1 \mathrm{~h}$ after an injection of LPS and $24.5 \pm 0.3 \mathrm{~h}$ after an injection of PHA (Parmentier et al. 1998; Smits et al. 1999). The 4-h delay was chosen for LPS because it corresponds to the peak of the cutaneous response following an injection of LPS in domestic fowls (Gallus gallus domesticus; Parmentier et al. 1998). Likewise, the $24 \mathrm{~h}$ delay corresponds to a peak of the cutaneous response to PHA in chicken (Parmentier et al. 1998) and house sparrows (Passer domesticus; Martin II et al. 2003).

After having measured swelling immune responses to LPS and PHA, we immediately brought one nestling per nest in the lab in the afternoon (day 0 ) and kept them at $24^{\circ} \mathrm{C}$ and 14:10 Light:Dark cycle singly in a similar nestbox as the one where they were reared in natural conditions (see also Piault et al. 2009). Due to space constraints we could not bring to the laboratory the other siblings that remained in their original nest. Despite we brought only one nestling per brood in the laboratory, all the nestlings of the brood were immune challenged for the purpose of another study on nestling immunocompetence (see Gasparini et al. 2009). To minimize variation in hunger level due to the fact that nestlings consumed different amounts of food during the last $24 \mathrm{~h}$ in natural conditions, we offered to all experimental individuals adult laboratory mice ad libitum until the next morning (08:00) when food treatments started (day 1). From day 1 to day 7 , nestlings were fed either ad libitum (i.e. they could eat as many laboratory mice as they wanted) or with a restricted diet (i.e. each nestling could eat only two laboratory mice per day). In each treatment, we added fresh laboratory mice in nest-boxes and removed non-eaten items at both 08:00 and 18:00 every day. Owlets could consume mice when they wanted since at that age they can swallow entire items or cut them into pieces before consumption. In natural conditions, nestlings consume between two and four small mammals per day (Galeotti 2001); main preys are field mice Apodemus sp. (body mass range: $22-45 \mathrm{~g}$ ) and bank voles Clethrionomys glareolus (body mass range: 15-25 g). Hence, two laboratory mice are below what nestlings would eat in the wild under favourable environmental conditions. Nestling body mass, tarsus length and wing length were measured to the nearest $0.1 \mathrm{~g}, 0.1 \mathrm{~mm}$ and $1 \mathrm{~mm}$ respectively on day 1 at 8:00. Nestling age, body mass, tarsus length, wing length and nestling rank in the age hierarchy in the natural brood did not differ between the four treatments at the start of the experiment (two-way ANOVA with food treatment and immune challenge as fixed factors plus their interaction, all $P$-values $>0.13$ ). However, note that because swelling immune response against LPS was measured $4 \mathrm{~h}$ after injection while swelling immune response against PHA was measured $24 \mathrm{~h}$ after injection and because all the nestlings were brought in the laboratory immediately after having measured the peak swelling immune response, PHA nestlings were 1 day younger than LPS nestlings when they were injected. Nineteen ( 9 females and 10 males) of the 38 PHA-injected nestlings were assigned to the ad libitum treatment and the 19 (9 females and 10 males) others to the restricted food treatment. Similarly, 21 LPS-injected nestlings were assigned to the ad libitum treatment (10 females and 11 males) and 21 to the restricted food treatment (12 females and 9 males). Males and females were randomly distributed among the four experimental groups (nominal logistic regression with nestling sex as the dependent variable, food and immune treatments as fixed factors plus their interaction, all $P$-values $>0.65$ ). Nestlings were returned to their original nest at day 7 in the morning after body mass, tarsus and wing length had been measured a second time. Thus, our experimental design allowed us to precisely follow the growth trajectory of nestlings between 21.7 and 28.7 ( $\mathrm{SE}=0.26)$ days of age, which coincides with the growth period just preceding fledging. Because body mass at fledging positively correlates with survival before dispersal in nestling tawny owls (Overskaug et al. 1999), selection may favour nestlings maintaining high growth rates before leaving the nest. Thus, here we used variation in growth rates just before fledging as a fitness proxy. To investigate the fate of the experimental owlets after having been released in the wild, from winter 2005 to 
spring 2010 we regularly controlled all the nest-boxes to capture adult owls, and in turn to gather data on offspring recruitment rate. Body mass at adulthood was measured to the nearest $1 \mathrm{~g}$.

\section{Ethical Note}

Time in the laboratory had no apparent negative consequence on nestling survival since young kept in captivity during 7 days were captured as adults in 2006-2010 as frequently $(17$ out of $80 ; 21.3 \%)$ as their siblings that stayed in their natural nest and were never brought to the laboratory (31 out of $205 ; 15 \%$; logistic regression with recruitment coded as 1 or $0, \chi^{2}=1.5, P=0.22$ ).

\section{Statistical Analyses}

To test whether food availability modulated differentially effect of LPS- and PHA-induced immune challenges on nestling body mass increments, we entered nestling body mass change over 6 days in the laboratory (body mass at day 7 - body mass at day 1) as the dependent variable in an ANCOVA with food treatment and immune challenge as fixed factors plus their interaction. Body mass at day 7 was entered as a covariate in these models to account for the fact that nestling growth trajectories may differ between small and large nestlings. We used body mass at day 7 rather than at day 2 to minimize regression to the mean effect (Kelly and Price 2005). A significant interaction between food treatment and immune challenge would show that variation in the relative cost of different immune challenges depends on food availability. To investigate the effect of immune challenges on nestling body mass change separately in the ad libitum and food-restricted treatment, we ran two separate ANCOVAs, one ANCOVA per food treatment. Within each ANCOVA model, immune challenge was entered as fixed factor and mean food consumption as a covariate to account for the large variance within each feeding treatment in the mean amount of mice consumed by each owlet during their stay of 6 days in the laboratory. We also investigated whether mounting a potent immune response can affect owlet body mass change by running ANCOVA models within each immune challenge. In these models, we entered owlet body mass change as the dependent variable, food treatment as a fixed factor, skin swelling response as a covariate, plus the interaction between food treatment and skin swelling response. Finally, to investigate potential long-term effects of our experiment on offspring recruitment rate (coded as 1/0) and body mass at adulthood, we used a logistic regression or an ANOVA, respectively, with food treatment and immune challenge as two factors plus their interaction. Statistical analyses were performed using JMP IN 7.0.0. Throughout the paper means are quoted $\pm \mathrm{SE}$, statistical tests are two-tailed and significance level set to 0.05 .

\section{Results}

Our immune challenges were efficient since nestlings mounted significant skin swelling response against both injected immunostimulants as measured by the change in skin thickness at the site of injection $4 \mathrm{~h}$ after LPS injection (Student's $t$-test comparing change in skin thickness with zero, $t_{41}=5.30, P<0.0001$; mean change in thickness: $0.18 \pm 0.03 \mathrm{~mm}$ ) and $24 \mathrm{~h}$ after PHA injection (Student's $t$-test comparing change in skin thickness with zero, $t_{37}=$ 8.24, $P<0.0001$; mean change in thickness: $0.46 \pm$ $0.06 \mathrm{~mm})$.

Our food treatment reduced by $46.6 \%$ the mean consumption of mice by food-restricted owlets (two-way ANOVA: effect of food treatment: $F_{1,76}=468.71$, $P<0.0001$ ), with owlets from the ad libitum treatment consuming on average $56.0 \pm 1.2 \mathrm{~g}$ per day (range: $38.7-75.5 \mathrm{~g}$ ) and food-restricted owlets eating $29.9 \pm$ $0.2 \mathrm{~g}$ per day (range: $27.0-31.8 \mathrm{~g}$ ). Note that although nestlings fed ad libitum decreased their food consumption between day $1 \quad(69.4 \pm 2.8 \mathrm{~g}$ per day) and day 7 $(48.3 \pm 2.1 \mathrm{~g}$ per day), the amount of food provided to food-restricted nestlings (i.e. $29.9 \pm 0.2 \mathrm{~g}$ per day) never reached the daily food requirement of nestlings within the ad libitum treatment. The mean consumption of mice by owlets was not influenced by immune challenges alone $\left(F_{1,76}=1.24, P=0.27\right)$ or in interaction with food treatment $\left(F_{1,76}=1.17, P=0.28\right.$; mean $\pm \mathrm{SE}$ consumption of LPS- vs. PHA-owlets within the ad libitum treatment: $57.3 \pm 1.5 \mathrm{~g}$ vs. $54.6 \pm 1.9 \mathrm{~g}$; LPS- vs. PHA-owlets within the food-restricted treatment: $29.9 \pm 0.2 \mathrm{~g}$ vs. $29.9 \pm 0.3 \mathrm{~g}$ ), thus indicating that immune challenges did not differentially modulate appetite.

Owlet body mass change over 6 days was significantly explained by the immune challenge in interaction with food treatments $\left(F_{1,75}=4.55, P=0.036\right)$. This ANCOVA model showed also that owlet body mass change was significantly explained by the food treatment alone $\left(F_{1,75}=36.09, P<0.001\right)$ but not by the immune challenge alone $\left(F_{1,75}=0.001, P=0.97\right)$, nestlings gaining more mass when fed ad libitum rather then with a restricted amount of food. Owlet body mass change was positively associated with their body mass at day 7 indicating that heavy nestlings at day 7 grew at faster rates than light ones $\left(F_{1,75}=4.65, P=0.034\right)$. ANCOVA models performed within each food treatment (with owlet body mass change as the dependent variable, owlet immune challenge as a factor, and owlet mean food consumption as a covariate) showed that the interaction between immune challenge and 


\section{A Ad libitum treatment}



\section{B Food restricted treatment}



Fig. 1 Change in body mass of tawny owl nestlings challenged with LPS or PHA in relation to their mean daily food consumption over 6 days. Owlets were raised over 6 days with an ad libitum food treatment (a) or a food-restricted treatment (b)

food treatment was explained by the fact that LPS-injected nestlings lost significantly more body mass than PHAinjected ones in the food-restricted treatment $\left(F_{1,37}=4.13\right.$, $P=0.049$; Fig. 1b), while there was no difference in daily change in body mass between LPS- and PHA-injected nestlings in the ad libitum treatment $\left(F_{1,37}=0.77\right.$, $P=0.38$; Fig. 1a). Owlet body mass change within each food treatment was significantly and positively associated with mean food consumption (all $P$-values $>0.001$ ) but not with body mass at day 7 (all $P$-values $>0.18$; body mass at day 7 was not included as a covariate in the final ANCOVA models performed within each food treatment). ANCOVA models performed within each immune challenge (with owlet body mass change as the dependent variable, owlet food treatment as a factor, and owlet mean skin swelling response and body mass as a two covariates) showed that a potent skin swelling response against LPS was associated with a reduction in body mass gain of nestlings in the ad libitum treatment but not in the food restricted treatment (interaction between food treatment and skin swelling: $F_{1,37}=7.61, P=0.009$; food treatment effect: $F_{1,37}=$ $23.54, P>0.001$; skin swelling response effect: $F_{1,37}=$ $8.99, P=0.005$; Fig. 2a). Owlet body mass change was no

\section{A LPS-injected treatment}

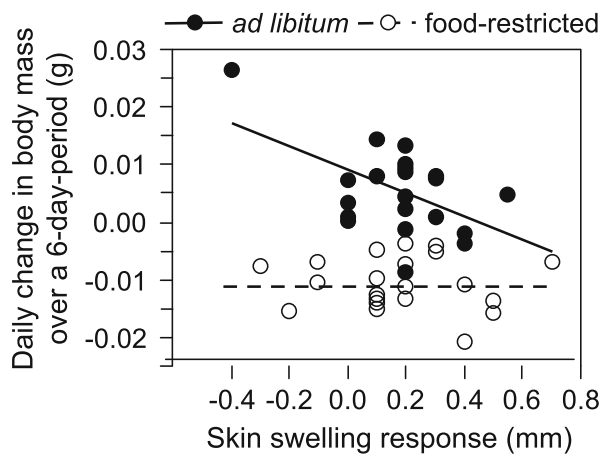

B PHA-injected treatment

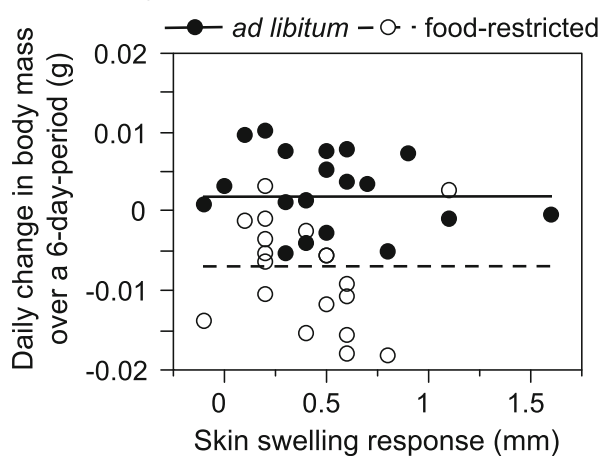

Fig. 2 Change in body mass over a 6-day period of tawny owl nestlings fed ad libitum or with a food-restricted diet in relation to their skin swelling response against an immunostimulant injected subcutaneously in the wing web. Owlets were injected with $20 \mu \mathrm{g}$ of LPS (a) or $10 \mu \mathrm{g}$ of PHA (b), and subsequently raised during 6 days under two different food regimes (ad libitum vs. food-restricted)

related with the magnitude of the skin swelling response against PHA whatsoever the food treatment (interaction between food treatment and skin swelling: $F_{1,33}=0.04$, $P=0.85 ;$ food treatment effect: $F_{1,33}=10.74, P=$ 0.003 ; skin swelling response effect: $F_{1,33}=1.51, P=$ 0.23 ; Fig. 2b). Owlet body mass change was significantly and positively associated with body mass at day 7 in the LPS-treatment $\left(F_{1,37}=7.89, P=0.008\right)$ but not in the PHA treatment $\left(F_{1,33}=2.58, P=0.12\right)$. Body mass change was similar between male and female owlets within each feeding treatment (all $P$-values $>0.55$ ) and within each immune challenge (all $P$-values $>0.08$ ). Thus, we excluded nestling sex from the final statistical models. Similar statistical analyses conducted on owlet wing growth and tarsus growth over the 6 experimental days revealed no significant effect of immune challenge alone or in interaction with food treatment on these two traits (all $P$-values $>0.26$; results not shown). Finally, neither food treatment nor immune challenge, alone or in interaction, had a significant effect on offspring recruitment rate in the study population after having been released in the wild (logistic regression: interaction between food 
treatment and immune challenge: $\chi^{2}=1.85, P=0.17$; food treatment effect: $\chi^{2}=0.08, P=0.78$; immune challenge effect: $\chi^{2}=1.31, P=0.25$ ) and on body mass at adulthood of the offspring recruited in the study population (ANOVA: interaction between food treatment and immune challenge: $F_{1,11}=0.35, P=0.57$; food treatment effect: $F_{1,12}=0.21, P=0.65$; immune challenge effect: $F_{1,12}=2.12, P=0.16$ ).

\section{Discussion}

In the present study, we tested the hypothesis that variation in the immunity cost to different types of immune challenge is exacerbated under conditions of low food availability. We investigated cost of immunity by measuring body mass change prior to fledging of nestling tawny owls injected with $10 \mu \mathrm{g}$ of PHA or $20 \mu \mathrm{g}$ of LPS, and subsequently raised during 6 days with an ad libitum diet or a food-restricted diet. In accordance with our prediction, we found in the food-restricted treatment that nestlings injected with $20 \mu \mathrm{g}$ of LPS lost more body mass than nestlings injected with $10 \mu \mathrm{g}$ of PHA, while no significant difference in body mass gain was observed between LPS- and PHAinjected nestlings in the ad libitum treatment. These findings suggest that variation in the relative cost of different types of immune challenge, due to host exposure to different immunostimulants, becomes manifest when food resources are limited (i.e. is condition-dependent). Interestingly, owlets mounting a potent skin immune response against LPS gained less body mass in the ad libitum treatment but not in the food-restricted treatment. There was no relationship between skin immune response against PHA and owlet change in body mass when fed ad libitum or with a food-restricted diet. These experimental and correlative findings suggest that mounting an immune response against LPS incur greater costs, in terms of body mass maintenance, than mounting an immune response against PHA, and that variation in the costs of two different types of immune challenge can be exacerbated under conditions of low food availability. Hereafter, we first discuss physiological factors that may account for the differential costs of LPS and PHA immune responses. Second, we discuss how variation in indirect costs of immunity (due to host exposure to different parasites or different doses of the same parasite) can shape the evolution of host-parasite interactions. Finally, because immune responses incur probably benefits and costs not only for hosts mounting an immune response but also for parasites eliciting an immune response, we also discuss how a better understanding of indirect costs of parasitism may help gain insights on the evolution of host resistance strategies and parasite exploitation strategies.
Differential Costs of LPS- and PHA-Immune Responses

At least two non-mutually exclusive hypotheses may account for greater effects of LPS immune challenges on nestling body mass compared to PHA immune challenges. First, PHA molecules might be less immunogenic than LPS molecules. PHA leads to the agglutination of erythrocytes at the point of inflammation after injection (i.e. is an erythroagglutinin; Yachnin and Svenson 1972). Thus, one hypothesis is that PHA molecules associated with erythrocytes may not be available to recruit T-lymphocytes and other leukocytes (Mayringer et al. 2000), resulting in relatively weak immune and acute-phase responses. Accordingly, at the cellular level it has been found that whole blood cultures (i.e. with erythrocytes) treated in vitro with PHA contain lower concentrations of pro-inflammatory cytokines than peripheral blood mononuclear cell cultures (i.e. without erythrocytes) (Mayringer et al. 2000). As a result, at the organismal level in vivo PHA challenge induces short-term immune and acute-phase responses characterized by a low stress response and minor systemic changes in body mass (Merino et al. 1999; Martin II et al. 2003) while LPS challenge induces strong immune and acute phase responses including a significant stress response and elevated systemic metabolism (Klasing et al. 1987; Cheng et al. 2004). In line with our results, previous studies have reported costs of LPS challenges on body mass maintenance of various adult and nestling species (e.g. Bonneaud et al. 2003; Cheng et al. 2004; Bertrand et al. 2006), whereas PHA challenges has been reported to have little or no effect on body mass maintenance despite being associated with an increase in resting metabolic rate (Merino et al. 1999; Martin II et al. 2003). Stronger indirect costs of LPS versus PHA challenges on body mass maintenance is further demonstrated by the fact that nestlings fed ad libitum and mounting a potent response against LPS gained less mass than individual fed ad libitum but mounting a relatively weak immune response against LPS (no similar relationship was detected for PHA). A second hypothesis for the stronger effect of LPS versus PHA challenges on nestling body mass growth is that we injected a dose of LPS two times greater than the dose of PHA. Previous studies suggest, however, that this hypothesis is unlikely. Indeed, Martin II et al. (2003) did not find any significant effect of PHA on body mass in adult house sparrows (Passer domesticus) although they injected a PHA dose 10 times greater than the dose we injected in nestling tawny owls $(100 \mu \mathrm{g}$ vs. $10 \mu \mathrm{g})$, and house sparrows are about 10 times lighter than owlets used in our study ( $28 \mathrm{~g}$ vs. $300 \mathrm{~g}$ ). By contrast, $10 \mu \mathrm{g}$ of LPS resulted in significant body mass loss and sickness symptoms in house sparrows (Bonneaud et al. 2003). Future 
studies involving a larger diversity in the types of immune challenge should help identifying which immune branches imposed high indirect costs of parasitism, and in turn might help explaining the observed variance in immune responses (Schmid-Hempel 2003).

\section{Effect of Indirect Costs of Parasitism on Host Resistance Strategies}

Our results point out that some parasites may incur greater fitness costs to their hosts not only by exploiting their hosts at faster rates (i.e. greater direct costs of parasitism; Frank 1996) but also by eliciting more costly immune responses (i.e. greater indirect costs of parasitism; Graham et al. 2005; Day et al. 2007). Thus, a first prediction is that, to be evolutionary stable, the production of costly immune responses should be proportional to direct costs inflicted by parasites, with hosts mounting costly immune responses against highly virulent parasites. Yet, because the cost of immunity can be exacerbated when food resources are low, a second prediction is that hosts should down-regulate their immune responses when resources are scarce. Accordingly, it is frequently observed that hosts in poor condition mount weaker immune responses than hosts in good condition (Chandra 1996; Sheldon and Verhulst 1996). However, to which extent this reduction in host immunocompetence when resources are limited is a host adaptive response or reflects an environmental constraint is unclear. To shed light on this question, experiments testing simultaneously the effects of host nutritive status and immunocompetence on host fitness and parasite fitness are needed as recently carried out in another bird species, the Alpine swift (Apus melba) (Bize et al. 2008). Knowledge of the costs and benefits of mounting an immune response under variable environmental conditions may provide valuable guidance for drug medication in veterinary and human medicine.

\section{Effect of Indirect Costs of Parasitism on Parasite Exploitation Strategies}

Costs of mounting an immune response may alter not only host fitness but also parasite fitness. Indeed, there is growing evidence from the biomedical literature that excessive immune responses can cause damage to host tissues or even host death (i.e. immunopathology), and that these immune-mediated modification of host phenotype can favour or impede parasite lifetime transmission between hosts (Graham et al. 2005). For example, immunopathological necrosis of the lung caused by tuberculosis enhances the transmission of bacilli (Kaushal et al. 2002), whereas uncontrolled immune responses to malaria infection lead to premature host death and reduce parasite lifetime transmission potential (Long et al. 2008). Thus, to maximize fitness parasites may either trigger extreme or reduced immune responses depending on whether immuneinduced host illness favours or impedes lifetime transmission potential, respectively (Graham et al. 2005). Yet, because host immune responsiveness is sensitive to resource levels (Chandra 1996), one hypothesis is that host susceptibility to parasite manipulation of host immune system can dramatically differ between resource-poor and resource-rich environments in interaction with parasite exploitation strategy. Parasites with immunopathologyenhanced fitness might do best in resource-rich environments and parasites with immunopathology-reduced fitness in resource-poor environments. Effect of environmental factors on parasite virulence is still poorly understood, and the importance of parasite manipulation of host immunity remains to be tackled in details (Graham et al. 2005; Zaccone et al. 2008).

Acknowledgments This study was supported by the Swiss National Science Foundation (grant no. PPOOA-102913 and 31003A-120517 to AR, PPOOA-109009 to PB and PBLAP3-124279 to RP) and the Roche Research Foundation (grant no. Mkl/stm 14-2008 to RP). The experiment was approved by the veterinary services of Canton de Vaud (licence no. 1508) and birds were ringed under the legal authorization of the Swiss Agency for the Environment, Forests and Landscape.

\section{References}

Amat, J. A., Aguilera, E., \& Visser, G. H. (2007). Energetic and developmental costs of mounting an immune response in greenfinches (Carduelis chloris). Ecological Research, 22, 282-287.

Bertrand, S., Criscuolo, F., Faivre, B., \& Sorci, G. (2006). Immune activation increases susceptibility to oxidative tissue damage in zebra finches. Functional Ecology, 20, 1022-1027.

Bize, P., Jeanneret, C., Klopfenstein, A., \& Roulin, A. (2008). What makes a host profitable? Experimental evidence that parasites balance host nutritive resource against immunity. American Naturalist, 171, 107-118.

Bonneaud, C., Mazuc, J., Gonzalez, G., Haussy, C., Chastel, O., Faivre, B., et al. (2003). Assessing the cost of mounting an immune response. American Naturalist, 161, 367-379.

Brzek, P., \& Konarzewski, M. (2007). Relationship between avian growth rate and immune response depends on food availability. Journal of Experimental Biology, 210, 2361-2367.

Chandra, R. K. (1996). Nutrition, immunity and infection: From basic knowledge of dietary manipulation to practical application of ameliorating suffering and improving survival. Proceedings of the National Academy of Sciences of the United States of America, 93, 14304-14307.

Cheng, H. W., Freire, R., \& Pajor, E. A. (2004). Endotoxin stress responses in chickens from different genetic lines. 1. Sickness, behavioral, and physical responses. Poultry Science, 83, 707-715.

Dantzer, R., Bluthe, R. M., Gheusi, G., Cremona, S., Laye, S., Parnet, P., et al. (1998). Molecular basis of sickness behavior. Annals of the New York Academy of Sciences, 856, 132-138. 
Day, T., Graham, A. L., \& Read, A. F. (2007). Evolution of parasite virulence when host responses cause disease. Proceedings of the Royal Society of London Series B: Biological Sciences, 274, 2685-2692.

Fair, J. M., Hansen, E. S., \& Ricklefs, R. E. (1999). Growth, developmental stability and immune response in juvenile Japanese quails (Coturnix coturnix japonica). Proceedings of the Royal Society of London Series B: Biological Sciences, 266, $1735-1742$.

Frank, S. A. (1996). Models of parasite virulence. The Quarterly Review of Biology, 71, 37-78.

Galeotti, P. (2001). Tawny owl Strix aluco. Birds of the Western Palaearctic Update, 3, 43-77.

Gasparini, J., Piault, R., Bize, P., \& Roulin, A. (2009). Synergetic and antagonistic interaction between different branches of the immune system is related to melanin-based coloration in nestling tawny owls. Journal of Evolutionary Biology, 22, 2348-2353.

Graham, A. L., Allen, J. E., \& Read, A. F. (2005). Evolutionary causes and consequences of immunopathology. Annual Review of Ecology, Evolution, and Systematics, 36, 373-397.

Jacot, A., Scheuber, H., \& Brinkhof, M. W. G. (2004). Costs of an induced immune response on sexual display and longevity in field crickets. Evolution; International Journal of Organic Evolution, 58, 2280-2286.

Kaushal, D., Schroeder, B. G., Tyagi, S., Yoshimatsu, T., Scott, C., Ko, C., et al. (2002). Reduced immunopathology and mortality despite tissue persistence in a Mycobacterium tuberculosis mutant lacking alternative sigma factor, SigH. Proceedings of the National Academy of Sciences of the United States of America, 99, 8330-8335.

Kelly, C., \& Price, T. D. (2005). Correcting for regression to the mean in behaviour and ecology. American Naturalist, 166, 700-707.

Klasing, K. C. (1988). Nutritional aspects of leukocytic cytokines. Journal of Nutrition, 118, 1436-1446.

Klasing, K. C., Laurin, D. E., Peng, R. K., \& Fry, D. M. (1987). Immunologically mediated growth depression in chicks-influence of feed-intake, corticosterone and interleukin-1. Journal of Nutrition, 117, 1629-1637.

Kobasa, D., Jones, S. M., Shinya, K., Kash, J. C., Copps, J., Ebihara, H., et al. (2007). Aberrant innate immune responses in lethal infection of macaques with the 1918 influenza virus. Nature, 445, 319-323.

Long, G. H., Chan, B. H. K., Allen, J. E., Read, A. F., \& Graham, A. L. (2008). Experimental manipulation of immune-mediated disease and its fitness costs for rodent malaria parasites. $B M C$ Evolutionary Biology, 8, 128.

Martin, L. B., I. I., Han, P., Lewittes, J., Kuhlman, J. R., Klasing, K. C., \& Wikelski, M. (2006). Phytohemagglutinin-induced skin swelling in birds: Histological support for a classic immunoecological technique. Functional Ecology, 20, 290-299.

Martin, L. B., I. I., Scheuerlein, A., \& Wikelski, M. (2003). Immune activity elevates energy expenditure of house sparrows: A link between direct and indirect costs? Proceedings of the Royal Society of London Series B: Biological Sciences, 270, 153-158.

Mayringer, I., Reindl, M., \& Berger, T. (2000). A critical comparison of frequently used methods for the analysis of tumor necrosis factor-alpha expression by human immune cells. Journal of Immunological Methods, 235, 33-40.

Merino, S., Martinez, J., Moller, A. P., Sanabria, L., De Lope, F., Perez, J., et al. (1999). Phytohaemagglutinin injection assay and physiological stress in nestling house martins. Animal Behaviour, 58, 219-222.
Mire-Sluis, A. R., Wickremasinghe, R. G., Hoffbrand, A. V., Timms, A. M., \& Francis, G. E. (1987). Human T lymphocytes stimulated by phytohaemagglutinin undergo a single round of cell division without a requirement for interleukin- 2 or accessory cells. Immunology, 60, 7-12.

Moret, Y., \& Schmid-Hempel, P. (2000). Survival for immunity: The price of immune system activation for bumblebee workers. Science, 290, 1166-1168.

Munford, R. S. (2006). Severe sepsis and septic shock: The role of gram-negative bacteremia. Annual Review of Pathology: Mechanisms of Disease, 1, 467-496.

Nossal, G. J. (1983). Cellular mechanisms of immunologic tolerance. Annual Review of Immunology, 1, 33-62.

Overskaug, K., Bolstad, J. P., Sunde, P., \& Oien, I. J. (1999). Fledgling behavior and survival in northern tawny owls. Condor, $101,169-174$.

Parmentier, H. K., De Vries Reilingh, G., \& Nieuwland, M. G. (1998). Kinetic and immunohistochemical characteristics of mitogen-induced cutaneous hypersensitivity in chickens selected for antibody responsiveness. Veterinary Immunology and Immunopathology, 66, 367-376.

Piault, R., Gasparini, J., Bize, P., Jenni-Eiermann, S., \& Roulin, A. (2009). Phaeomelanin-based coloration and the ability to cope with variation in food supply and parasitism. American Naturalist, 174, 548-556.

Roulin, A., Ducret, B., Bize, P., Piault, R., \& Ravussin, P.-A. (2008). Régime alimentaire de la Chouette hulotte Strix aluco en Suisse romande de 1986 à 2007. Nos Oiseaux, 55, 149-156.

Saladin, V., Ritschard, M., Roulin, A., Bize, P., \& Richner, H. (2007). Analysis of genetic parentage in the tawny owl (Strix aluco) reveals extra-pair paternity is low. Journal of Ornithology, 148, 113-116.

Schmid-Hempel, P. (2003). Variation in immune defence as a question of evolutionary ecology. Proceedings of the Royal Society of London Series B: Biological Sciences, 270, 357-366.

Sheldon, B. C., \& Verhulst, S. (1996). Ecological immunology: Costly parasite defences and trade-offs in evolutionary biology. Trends in Ecology \& Evolution, 11, 317-321.

Skidmore, B. J., Chiller, J. M., Morrison, D. C., \& Weigle, W. O. (1975). Immunological properties of bacterial lipopolysaccharide (Lps): Correlation between mitogenic, adjuvant, and immunogenic activities. Journal of Immunology, 114, 770-775.

Smits, J. E., Bortolotti, G. R., \& Tella, J. L. (1999). Simplifying the phytohaemagglutinin skin-testing technique in studies of avian immunocompetence. Functional Ecology, 13, 567-572.

Stjernman, M., Raberg, L., \& Nilsson, J.-A. (2008). Maximum host survival at intermediate parasite infection intensities. PLOS ONE, 3 , e3463.

Tella, J. L., Lemus, J. A., Carrete, M., \& Blanco, G. (2009). The PHA test reflects acquired $\mathrm{T}$-cell mediated immunocompetence in birds. PLoS ONE, 3, e3295.

Yachnin, S., \& Svenson, R. H. (1972). Immunological and physicochemical properties of mitogenic proteins derived from Phaseolus vulgaris. Immunology, 22, 871-883.

Zaccone, P., Burton, O. T., \& Cooke, A. (2008). Interplay of parasitedriven immune responses and autoimmunity. Trends in Parasitology, 24, 35-42.

Zuk, M., \& Stoehr, A. M. (2002). Immune defense and host life history. American Naturalist, 160, S9-S22. 